\title{
LA GÉNESIS DE LA EDICIÓN DE LOS COMMENTARII DE CÉSAR DE VENECIA, 1511: ¿EMENDATIO ORIGINAL O MERA COPIA? ${ }^{1}$
}

\author{
Antonio Moreno HeRnándeZ \\ UNED. Madrid \\ anmoreno@flog.uned.es
}

\section{RESUMEN}

La edición de los Commentarii de César impresa en Venecia en 1511 por Augustinus de Zannis es, en apariencia, un fruto de la práctica filológica habitual de la época, la emendatio humanística, a tenor del título y el colofón de la obra y del prefacio de Lucas Olchinensis, en el cual éste declara que ha preparado la edición a base de enmendar las numerosas corrupciones de la tradición anterior. Sin embargo el análisis crítico de la edición veneciana y su colación con las ediciones precedentes permite comprobar que el texto de 1511 depende completamente de la edición de Florencia 1508 impresa por Philippus Giunta y preparada por Lucas Robia, mediante la reproducción mimética de las variantes de su modelo; pero la edición veneciana ha intentado disimular su dependencia presentando el aspecto de una edición incunable e introduciendo diversas divergencias en la composición que aparentemente la alejan de su modelo.

Palabras Clave: Commentarii; Julio César; Augustinus de Zannis; Lucas Olchinensis; emendatio.

\section{ABSTRACT}

The edition of Caesar's Commentarii printed in Venice in 1511 by Augustinus de Zannis is outwardly the result of the philological practice of the time, the humanistic

${ }^{1}$ Este artículo se inscribe dentro del Proyecto de Investigación HUM2005-00442, subvencionado por la Dirección General de Investigación, del Ministerio de Educación y Ciencia. 
emendatio, according to the title and the colophon of this work and the Praefatio of Lucas Olchinensis, where he declares that he has prepared this edition by means of the emendation of many corruptions transmitted by the previous tradition. However, the critical analysis of the venetian edition and its collation with the earlier editions let us prove that this text of 1511 is completely based on the florentine edition of 1508, printed by Philippus Giunta and prepared by Lucas Robia, through the mimetic reproduction of its model; but the venetian edition seems to have made and attempt to hide its dependence by showing the aspect of an incunable edition and introducing some differences in the composition that seemingly keep this edition away from its model.

Key Words: Commentarii; Iulius Caesar; Augustinus de Zannis; Lucas Olchinensis; emendatio.

\section{INTRODUCCIÓN}

En los primeros años del s. XVI se produce en Italia una extraordinaria efervescencia editorial en torno a los Commentarii de César, alentada por el ambiente de impresores y editores humanistas que en apenas diez años propicia la aparición de cuatro ediciones de estas obras: la edición boloñesa de $1504^{2}$, la florentina de $1508^{3}$ y las dos venecianas de $1511^{4}$ y $1513^{5}$.

Todas estas ediciones tienen, aparentemente, un factor común: la intervención en las labores de preparación del texto de humanistas de la época versados en los textos clásicos, que dejan constancia en los prefacios de sus ediciones de la labor que llevan a cabo dentro de los presupuestos de las técnicas filológicas de la época, que giran fundamentalmente en torno a la práctica de la emendatio de la tradición anterior6.

${ }^{2}$ Con la intervención de Philippus Beroaldus, en el taller de Benedictus Hector, Commentarii Caesaris, recogniti per Philippum Beroaldum... Impressit Bononiae Benedictus Hectoris (Bolonia, abril de 1504); editada depués por Balthazar Gabianus en Lión, en 1508 y en 1512.

${ }^{3}$ Con la participación de Lucas Robia, para la prensa de Philippus Giunta, Commentaria Caesaris, edente Luca Robia. Impressum Florentiae, opera... Philippi Giuntae (Florencia, abril de 1508).

${ }^{4}$ Salida de las prensa de Augustino de Zannis, Caii Iulii Caesaris commentaria. Venetiis: per Augustinum de Zannis de Portesio, con la intervención de Lucas Olchinensis (Venecia, agosto de 1511).

5 Debida a Giovanni Giocondo, en las prensas de Aldo Manuzio (Venecia, noviembre de 1513).

${ }^{6}$ Sobre la noción y las modalidades de emendatio realizada específicamente por los primeros editores de impresos incunables y postincunables, así como la distancia entre la teoría y la práctica real en sus ediciones, hay que tener en cuenta el estudio fundamental de E.J. Kenney, The Classical Text. Aspects of Editing in the Age of the Printed Book, Berkeley - Los Ángeles - Londres, 1974, pp. 21-46 y 47-74; y en concreto sobre el proceso de elaboración de estas primeras ediciones, cf. A Severyns, Texte et apparat: histoire critique d' une tradition imprimée, Bruselas, 1962; para el contexto en el que se desenvuelve la nueva cultura de la imprenta y la relación entre los editores y los impresores en la preparación de los textos, cf. B. Richardson, Print Culture in Renaissance Italy, Cambridge, 1994 (en particular pp. 64-78 para la Venecia de 1501 a 1530); sobre el sentido de los términos que utilizan los humanistas en torno a la intervención en el texto, cf. también S. Rizzo, Il lessico filologico degli umanisti, Roma, 1984 (1973), pp. 243-300; así como los estudios sobre «Critica congeturale e testi umanistici (I, II y III)», en A. Perosa, Studi di filologia umanistica, a cura di P. Viti, Roma, 2000, vol. 2 (II. Il Quattrocento fiorentino), pp. 9-40. 
Este artículo tiene por objeto el análisis de una de estas primeras obras postincunables: la edición de Venecia 1511 (que denominaremos z $)^{7}$, impresa en los talleres de Augustinus de Zannis de Portesio $^{8}$ y de la que se conservan dos ejemplares en España ${ }^{9}$; desde Cosenza se considera habitualmente que «these 'Commentaria' were edited by Lucas Panetius» ${ }^{10}$, el humanista conocido también como Lucas Olchinensis ${ }^{11}$.

Augustinus de Zannis de Portesio ${ }^{12}$, impresor de clásicos latinos ${ }^{13}$ e italianos ${ }^{14}$ en folio, desarrolló su actividad en Venecia entre 1509 y $1528^{15}$, y estaba emparentado con otro célebre editor de la generación anterior, Bartholomeus de Zannis (1486-1515) con el que compartió la actividad en el taller durante algún tiempo.

Esta edición de los Commentarii se presenta, de acuerdo con los hábitos nada infrecuentes en la época, como un texto depurado de los errores de la tradición anterior gracias a la labor de un editor cultivado. Ya en el propio título que encabezaba la edición se insiste en la intervención a la que se ha sometido el texto de César:

Caii Iulii Caesaris invictissimi imperatoris Comentaria seculorum iniuria antea difficilia et valde mendosa. nunc primum a viro docto expolita et optime recognita.

Igualmente en el colofón se desarrolla la misma idea:

Habes lector peritissime cōmètaria ante impressorum iniuria trunca: et mendosa: Nunc autem a viro docto optime recognita: et in pristinum candorem redacta: Impressa mira diligentia Venetiis: per Augustinū de Zannis de Portesio (f. $\mathrm{p}_{6 \mathrm{v}}$ )

7 Caii Iulii Caesaris commentaria (Venetiis: per Augustinum de Zannis de Portesio, 1511 die XVII Augusti). Edición de referencia: Roanne, Médiathèque Municipale, R Fol 124, AN.

${ }^{8}$ También documentado con las siguientes variantes: Augustinus de Zannis; Augustinus de Zanis; Augustino de Zanni; Agostino Zani; Agostin da Portese; Augustino de Zani; Augustinus de Zannis de Portesio.

${ }^{9}$ Los dos se encuentran en Cáceres, Biblioteca Pública «A. Rodríguez Moñino y María Brey», sign. 1/4557 (CCPBE000455063-3); y sign. 1/8888(1) (CCPBE000456367-0). Cf. Apéndice.

${ }_{10}$ M. E. Cosenza, Biographical and bibliographical dictionary of the italian humanists, 6 vols., Boston, 1962-67, pp. 134-135.

11 A tenor de la dedicatoria de Lucas Olchinenis a Rosello de Rosellis que encabeza la obra, cf. pp. 25-26.

12 También documentado con las siguientes variantes: Augustinus de Zannis; Augustinus de Zanis; Augustino de Zanni; Agostino Zani; Agostin da Portese; Augustino de Zani; Augustinus de Zannis de Portesio.

13 Además del Corpus Caesarianum editado en 1511 y de nuevo en 1517, Augustinus de Zannis imprimió las Heroidas y otras obras de Ovidio (octubre de 1510), un Lucano (junio de 1511) y posteriormente, las Tusculanas de Cicerón (1516) y las obras de Virgilio (1519) —ésta última, a instancias de L. Antonio Giunta—, así como otras obras humanísticas, como el célebre tratado musical de Franchino Gaffurio, Practica musicae (1512).

${ }^{14}$ Entre sus obras en italiano, editó el Canzionere de Petrarca (1515) — dependiente del de Bembo- y el Decameron de Boccaccio (1518), basado en la edición florentina de 1516, cf. B. Richardson, Print Culture in Renaissance Italy, op. cit., pp. 55 y 208.

${ }^{15}$ G. Borsa. Clavis typographorum librariorumque Italiae: 1465-1600, Baden-Baden - Budapest, 1980, vol. II, p. 95; F. J. Norton, Italian Printers 1501-1520, Londres, 958, pp. 162-163; F. Ascarelli, M. Menato, La Tipografia del '500 in Italia, Florencia, 1989, p. 340; E. Sandal (ed.), Atti del Convegno Internazionale «I primordi della stampa a Brescia: 1472-1511», Padua, 1986; H. M. Adams, Catalogue of Books Printed on the Continent of Europe, 1501-1600, in Cambridge Libraries, Londres, 1967, p. 219 (edición de 1517, n. ${ }^{\circ} 28$ ); Index Aureliensis. Tertia Pars, Tomus III: Clavis typographorum librariorumque saeculi sedecimi, Baden Baden, 1992; Short-title catalogue of books printed in Italy and of Italian books printed abroad 1500-1600, Boston, 1970, vol. I, p. 320; P. Kristeller, Die italienischen Buchdrucker- und Verlegerzeichen bis 1525, Strassburg 1893 (reimpreso en Mansfield Center CT, 2000); E. Pastorello, Tipografi, editori, librai a Venezia nel secolo XVI, Florencia, 1924; F. Ascarelli, La tipografia cinquecentina italiana. Florencia, 1953, p. 174. 
Esta forma de presentar la obra a través de los elementos paratextuales responde sin duda al propósito de atraer la atención de los potenciales lectores, dentro de un entorno de gran competencia entre los editores italianos. En el caso de Augustinus de Zannis, este tratamiento de títulos y colofones es común a otras ediciones de clásicos latinos a lo largo de toda su trayectoria, como ocurre con las Heroidas y el Ibis de Ovidio de 1510, cuyo título y colofón recoge los mismos tópicos que la edición de César, insistiendo en la intervención de reputados eruditos, en términos prácticamente idénticos (expolitae... recognitae):

Epistole Heroides Ouidii: antea: impressorum omnium uitio: superuacaneae: mendosae: $\&$ difficiles. Nunc uero temporis a uiro docto expolitae: recognitae ... Scribentibus Antonio Volsco: \& Vbertino Crescentinate. In Ibim autem domitio Calderino: \& Christophoro Zaroto uiris eruditissimis.

Y con un colofón similar:

Impraessum Venetiis, mira diligentia per Augustinum de Zannis de Portesio, 1510 die XXV Octobris.

De hecho, en la misma época en la que imprime la edición de César, Ausgustinus de Zannis lleva a cabo otra de Lucano, que sale a la luz en junio de 1511, y que contiene una declaración bastante similar sobre la corrección del texto de la edición en el título de la obra:

Annei Lucani Bellorum civilium scriptoris accuratissimi Pharsalia antea temporum iniuria difficilis ac mendosa novissime autem a viro docto expolita [et] apprime plusq[ue] bis mille locis eme[n]data scribe[n]tibus Ioanne Sulpitio [et] Omnibono Vicentino ... ; additis isup [er] de novo grecis que ubiq[ue] deera[n] t necno[n] utilissimis apostillis una cum figuris ... itidemq[ue] alphabetica tabula ... - Impressum Venetiis: per Augustinum de Zanis de Portesio: impensis ... Melchioris Sessae, 1511 die IIII me[n] sis Iunii.

En febrero de 1516 sale a la luz la edición de las Tusculanae, en cuyo colofón se hace hincapié en la labor enmendadora del propio Lucas Olchinensis:

Finiunt Tusculanae quaestiones ... per Lucam Olchinensem artium et legum proffesorem accuratissime recognitae: cunctisque mendis expurgatae: Impressaeque Venetiis summa diligentia per Augustinum de Zannis de Portesio. Anno domino .M.D.XVI. Die .XV. Februarii.

Este mismo tenor se mantiene en otras ediciones posteriores de clásicos latinos, como en las obras de Virgilio, impresas en 1519, cuyo título reza así:

Vergilius cum commentariis. Opera Vergiliana antea corrupta et mendosa, nunc vero multorum exemplarium collatione in integrum restituta, docte et familiariter exposita ${ }^{16}$.

${ }^{16}$ Con la diferencia de que a continuación explicita los comentarios y anotaciones de los que se ha servido: Docte quidem Bucolica et Georgica a Seruio, Donato, Mancinello et Probo nuper addito, cum adnotationibus Beroaldinis. Aeneis vero ab iisdem praeter Mancinellum et Probum, et ab Augustino Datho in eius principio. Opusculorum praeterea quidam ab Domitio Calderino, familiariter vero omnia tam opera quam opuscula ab Iodoco Badio Luceno ... Omnia quidem tam Bucolica, Georgica, opusculorumque nonnulla et Aeneis, quam tertiusdecimus a Mappheo Vegio liber, expolitissimis figuris et imaginibus illustrata. 


\section{EL PREFACIO DE LUCAS OLCHINENSIS}

A la edición antecede un prefacio de Lucas Olchinensis ${ }^{17}$ dedicado a Rosello de Rosellis de Padua ${ }^{18}$. Lucas se presenta a si mismo como artium et legum professor y recoge él, junto al elogio a Rosello, algunas avatares de la preparación de la edición. Lucas Olchinensis colaboró con de Zannis en la edición de otras obras clásicas (como las Tusculanarum quaestionum liber, Venecia 1516, que incluían los comentarios de Philippus Beroaldus), así como de diversos tratados jurídicos con otros editores ${ }^{19}$.

La dedicatoria de Lucas Olchinensis, cargada de los tópicos habituales entre los humanistas de la época, ofrece algunos datos relevantes sobre el planteamiento de la edición:

Lucas comienza revelando la intensa dedicación que ha consagrado a la corrección (corrigendos) de los Commentarii, que ha llevado a término y que ahora presenta a la consideración de Rosello:

Caesaris invictissimi imperatoris commentarios: quos incredibili ardore corrigendos susceperam Roselle doctissime: deo propitio ad umbiculum perduxi. In quibus quantum laboris: et lucubrationis impenderim: tibi praecipue... arbitrandum relinquimus.

A continuación propone a Rosello que compare su edición con las editadas hasta el momento para que compruebe él mismo la utilidad que para los estudiosos tiene su labor, ante la gran cantidad de errores y faltas de los textos que circulaban en la época:

Si enim, mi Roselle, omnes recentiores textus: qui in hunc usque diem decussi sunt: huic nostro conferre volueris (nisi ego quoque pari fraude deceptus sim) liquido apparebit: quam omnibus studiosis profecerim. Legebantur quidem in tali ac tanto opere errores et soloecismi quam plurimi.

Lucas introduce aquí el tópico de la comparación de su obra con ediciones anteriores, muy común en los prefacios de las ediciones humanísticas, lo que le permite subrayar los defectos de las anteriores, y apunta seguidamente algunos datos sobre el procedimiento que ha seguido para realizar su edición:

Nos vero collatis multis exemplaribus: Beroaldi f. romani elloquii principis .M. Antonii: et aliorum trium eruditissimorum virorum: Caesarem ipsum: totius orbis monarcham. Qui (ut Quintilianus inquit) si tantum foro vacasset: nulus alius contra Ciceronem

17 También conocido por Luca Olchinense, y, sobre todo como Lucas Panetius, cf. M E. Cosenza, Biographical and bibliographical dictionary of the italian humanists, 6 vols., Boston, 1962-67, p. 134-135.

${ }_{18}$ Rosello de Rosellis es el hijo de un célebre jurisconsulto, Antonio de Rosellis de Aretio (1380-1466), al que Lucas alaba en la dedicatoria. Éste fue profesor de las universidades de Bolonia y de Padua, y alcanzó una cierta relevancia por los encargos diplomáticos que recibió del rey de Nápoles y del Papa Eugenio IV.

${ }^{19}$ Compedio de las obras jurídicas de Bartholomeus Cepolla, de Verona, Cautele... varii tractatus legum studiosis utilissimi, materias quotidianas \& practicabiles lucide complectentes... (impressa cum diligentia Joannis Crespini, Lyon, 1535); y el Repertorivm domini Philippi Decii, per Lucam Panetium Canonicum Olchinensem artium \& legum praeclarem doctorem fideliter concinnatum (impresa por Iacobus Giunta, Lyon 1544). 
nominaretur: mendosum, claudum: corruptum in pristinum candorem deduximus. Meruit utique Caesar: quem naturam ad hoc confinxisse crediderim: ne maiorem se aliquo modo videret: vel etiam a consumatissimo philosopho tot mendis expurgari. Sed cui nam potissimum has tumultuarias vigilias dedicarem: tu solus: mi Roselle: quoquo me verterem: occurrebas.

Lucas revela en este pasaje que ha llevado a cabo el cotejo de múltiples ejemplares de la obra (collatis multis exemplaris), entre los cuales no parece incluir manuscritos ${ }^{20}$ sino sólo ediciones, dado que a continuación se refiere a varios testimonios, como los de Philippus Beroaldus el joven (1453-1505 — autor de una edición publicada en Bolonia 1504, y posteriormente en Lyón 1508 y 1512)—, de un ‘Marco Antonio' —que quizá podría tratarse de algún ejemplar del humanista Marcus Antonius Sabellicus (14361506), director de la Biblioteca de San Marcos, y autor de unas annotationes a varios autores clásicos ${ }^{21}$ - y de otros tres eruditissimi viri, cuyo nombre no menciona; a partir de ellos Lucas trató de recuperar el esplendor del texto de César (in pristinum candorem deduximus), hasta entonces defectuoso (mendosum), cojo (claudum) y corrompido (corruptus), gracias a lo cual es posible limpiar de defectos el texto cesariano (mendis expurgari), tarea a la que consagra intensas veladas de trabajo (tumultuarias vigilias dedicarem).

Por último ofrece el texto fruto de sus correcciones - a las que denomina has emendationes - a Rosello, confiando en el provecho de su lectura:

Has igitur emendationes ad te transmittere volui: non quia earum lectioni te vaccare posse arbitrer. Video profecto aestus fluctuantis patriae tuae: sed ut omnia mea opera in familiares referantur. Si quid tamen per ocium licuerit: hos Caesaris commentarios legere non pigeat.

Esta presentación de la labor del editor no es ajena a muchos de los lugares comunes que manejan los humanistas de comienzos del s. XVI para realzar el valor de su edición entre los círculos de humanistas y de eruditos así como para provocar el interés por su adquisición entre los potenciales lectores.

\section{Dependencia teXtual de $\mathbf{z}$ Respecto a $\mathbf{f}$}

Sin embargo, estas manifestaciones del impresor y del editor de $\mathbf{z}$, que hacen gala de la labor filológica de corrección del texto, no parecen corresponderse con la realidad que la tradición del texto cesariano revela, pues el análisis del impreso veneciano y su colación con las ediciones anteriores del entorno humanístico italiano permite comprobar que el texto de $\mathbf{z}$ tiene por base una edición tres años anterior, impresa por Philippus Giunta

${ }^{20}$ Sobre la falta de interés por los manuscritos que muestran muchos editores postincunables, cf. M. Skole, Reading Sulpicia: Commentaries 1475-1990, Oxford, 2002, p. 31: «After the first printed editions appeared very few were interested in returning to the manuscripts until Scaliger in the Mid-Sexteenth Century».

${ }^{21}$ Marci Antonii Sabellici Annotationes veteres \& recentes: ex Plinio: Liuio: \& pluribus authoribus. Philippi Beroaldi Annotationes centum (Venecia 1502). Cf. M. Zorzi, La Libreria di San Marco. Libri, lettori, società nella Venezia dei Dogi, Milán, 1987. 
en Florencia en $1508^{22}$, que denominaremos $\mathbf{f}$ y que en realidad ha sido utilizada como la base — haciendo las veces de 'copia de impresor'"23 _, sobre la que se vuelve a componer el texto, pero en este caso sin introducir lecturas significativas que revelen una labor de emendatio apreciable.

En efecto, el análisis crítico de las lecturas de la edición florentina y su comparación con el comportamiento de la edición de 1511 permite comprobar que esta edición de Lucas Olchinensis, a pesar de tener un aspecto editorial muy distinto ${ }^{24}$, depende completamente de f, como demuestra la reproducción en aquélla de todas las peculiaridades textuales de la edición florentina. Ésta, a pesar de entroncar con la tradición impresa anterior, es el fruto de una labor de emendatio apreciable sobre el textus receptus de los incunables a través de distintos procedimientos, que reproduce $\mathbf{z}$ sistemáticamente $^{25}$ :

a) Mediante la integración de variantes de la tradición manuscrita de la parte alta del stemma frente a la lectura mayoritaria de las ediciones impresas anteriores y los mss. recentiores:

f se aparta de lecturas de las ediciones incunables basadas en recentiores para asumir variantes de la tradición antigua, como muestran los ejemplos que siguen, en los que f recoge una variante desconocida en la tradición impresa pero que responde a una variante de los mss. con mayor valor estemmático que resulta más plausible críticamente y que de hecho seleccionan los editores críticos modernos:

Gall. 1, 53,8 reductus est $\mathbf{f} \mathbf{z}$ adductus est edd. inc. b

Gall. 2, 1,2 ne omni pacata Gallia, ad eos exercitus noster adduceretur $\mathbf{f} \mathbf{z}$ ne omni pacata Gallia exercitus noster ad eos adduceretur edd. inc. b

Gall. 2, 4,4 ad id bellum $\mathbf{f} \mathbf{z}$ ad bellum edd. inc. $\mathbf{b}$

\footnotetext{
${ }^{22}$ Sobre el quehacer de Philippus como impresor y de toda la familia Giunta, cf. W. A. Pettas, The Giunti of Florence: merchant publishers of the sixteenth century (With a checklist of all books and documents publ. by the Giunti in Florence from 1497 to 1570, and with the texts of 29 documents, from 1427 to the 18th century, San Francisco), Rosenthal 1980; F. J. Norton, Italian Printers 1501-1520, Londres, 1958, pp. 29-31; cf. también los trabajos de F. Ascarelli, M. Menato, La Tipografia del' 500 in Italia, Florencia, 1989, 271-273; P. C. Marani, «I Giunti e il Rinascimento fiorentino», L'Esopo 1, 1979, pp. 51-55; C. Di Filippo Bareggi, «Giunta, Doni, Torrentino: tre tipografie fiorentine fra Reppublica e Principato», Nuova Rivista Storica 58, 1974, pp. 318-348. El nombre de nuestro impresor, Philippus Giunta, puede encontrarse también con estas otras formas latinas (Philippus Iuncta o Iunta) e italianas (Filippo Giunti; Filippo Gionti; Filippo Giunta).

${ }^{23}$ Cf. A. Severyns, Texts et Apparat, op. cit. pp. 17-68. A este original impreso que se reutilizaba con correcciones habitualmente tomadas de manuscritos se le ha conocido durante mucho tiempo como «manuscrit belge». Cf. A.E. Kenney, The Classical Text, op. cit., p. 26.

${ }^{24}$ Cf. Apartado 3.

25 Utilizamos las siguientes siglas para identificar a las ediciones:

A) Para las ediciones incunables (1469-1499): edd. inc. = consenso entre las ediciones incunables; $\mathbf{r}=$ Roma 1469 (Giovanni Andrea Bussi); v = Venecia 1471 (Nicolas Jenson); e = (1473 Strassbourg, Heinrich Eggestein / Esslingen, Conrad Fyner); m = Milán, 1477 (Antonius Zarotus); t = Treviso 1480 (Michael Manzolus); $\mathbf{v}^{\mathbf{s}}=$ Venecia 1482 (Octavianus Scotus); $\mathbf{v}^{\mathbf{r}}=$ Venecia 1490 (Theodorus de Ragazonibus); $\mathbf{v}^{\mathbf{p}}=$ Venecia 1494 (Philippus Pincius); $\mathbf{v}^{\mathbf{f}}=$ Venecia 1499 (Benedictus Fontana).

B) Para las ediciones de 1504 a 1513: b = Bolonia 1504 (impreso por Benedictus Hector, preparada por Philippus Beroaldus); $\mathbf{f}=$ Florencia 1508 (impreso por Philippus Giunta, preparada por Lucas Robia); $\mathbf{z}=$ Venecia 1511 (impreso por Augustinus de Zannis); a = Venecia 1513 (Editio Aldina, preparada por Giovanni Giocondo).
} 
Gall. 2, 4,7 imperium obtinuerit $\mathbf{f} \mathbf{z}$ regnum optinuerat edd. inc. $\mathbf{b}$

Gall. 2, 6,2 in murum lapides iaci $\mathbf{f} \mathbf{z}$ lapides in murum iaci $e d d$. inc. $\mathbf{b}$

Gall. 4, 1,8 lacte atque pecore $\mathbf{f} \mathbf{z}$ lacte ad pecore edd.inc. $\mathbf{b}$

Gall. 4, 3,1 esse laudem $\mathbf{f} \mathbf{z}$ laudem esse edd. inc. b

Gall. 4, 3,3 ampla atque florens $\mathbf{f} \mathbf{z}$ ampla ac florens edd. inc. $\mathbf{b}$

Civ. 1, 86,1 cognosci potuit $\mathbf{f} \mathbf{z}$ potuit cognosci edd. inc. $\mathbf{b}$

Civ. 1, 86,1 ultro praemium $\mathbf{f} \mathbf{z}$ ultro inde praemium $e d d$. in. $\mathbf{b}$

Civ. 1, 87,2 habuerunt $\mathbf{f} \mathbf{z}$ habuerant edd. inc. $\mathbf{B}$

Civ. 2, 1,2 ex Gallia atque Hispania $\mathbf{f} \mathbf{z}$ in Galliam atque Hispaniam $\mathbf{v} \mathbf{b}$ ad Galliam atque Hispaniam $\mathbf{r} \mathbf{t} \mathbf{v}^{\mathbf{s}} \mathbf{v}^{\mathbf{f}}$

Civ. 2, 2,2 balistis missi per quattuor $\mathbf{f} \mathbf{z}$ balistis per quattuor edd. inc. $\mathbf{b}$

Civ. 2, 2,5 altitudo muri $\mathbf{f} \mathbf{z}$ muri altitudo $e d d$. inc. $\mathbf{b}$

Civ. 2, 4,3 instructa classe $\mathbf{f} \mathbf{z}$ instructa acie $e d d$. inc. $\mathbf{b}$

Civ. 2, 5,1 diebus refecerat $\mathbf{f} \mathbf{z}$ refecerat diebus $\sigma$ edd. inc. $\mathbf{b}$

Civ. 2, 5,2 cohortatus $\mathbf{f} \mathbf{z}$ exhortatus edd. inc. b

Civ. 2, 7,1 conspectus $\mathbf{f} \mathbf{z}$ respectus edd. inc. b

Civ. 2, 7,3 et re cognita $\mathbf{f} \mathbf{z}$ hac re cognita $e d d$. inc. $\mathbf{b}$

Civ. 2, 7,3 urbs ab hostibus capta $\mathbf{f} \mathbf{z}$ urbs capta ab hostibus edd. inc. b

Civ. 2, 7,4 nihilo secius $\mathbf{f} \mathbf{z}$ nihilo segnius edd. inc. $\mathbf{b}$

Civ. 2, 8,1 fecerunt $\mathbf{f} \mathbf{z}$ fecerant edd. inc. $\mathbf{b}$

En todos estos casos, la edición veneciana de 1511 sigue la propuesta de f.

En otros casos, la variante de $\mathbf{f}$ que adopta $\mathbf{z}$ no está sustentada en el consenso de la tradición antigua sino en una parte de la misma:

- f sigue lecturas de la clase $\beta$ de Gall., que no había adoptado la tradición impresa anterior:

Gall. 1, 53,4 una Sueva natione, quam domo secum eduxerat $\beta, \mathbf{f} \mathbf{z}$ una Sueva natione, quam domo secum duxerat, $\alpha$ edd. inc. $\mathbf{b}$

Gall. 1, 53,5 hostium $\beta \mathbf{f} \mathbf{z}$ hostes $\alpha$ edd. inc. $\mathbf{b}$

Gall. 2, 3,1 primos civitatis suae miserunt $\beta \mathbf{f} \mathbf{z}$ primos civitatis miserunt $\alpha$ edd. inc. b

- f sigue lecturas de la clase $\alpha$ que no sigue la tradición anterior:

Gall. 2, 5,6 relinquit $\alpha$ reliquit $\beta$ edd. inc. b

Civ. 2, 1,3 a terra edd.inc. $\mathbf{b}$ ad terram $\sigma \mathbf{f} \mathbf{z}$

Civ. 2, 6,1 accideret $\mathbf{t} \mathbf{v}^{\mathbf{s}} \mathbf{v}^{\mathbf{f}}$ acciderit $\mathbf{v} \mathbf{b} \quad$ acciderat $\beta \mathbf{f} \mathbf{z}$

b) A través de la adopción de correcciones con base en la tradición incunables o en parte de ella. En ocasiones $\mathbf{f}$ y $\mathbf{z}$ siguen la lectura de una edición incunable, sobre todo de la de 1499:

Gall. 4, 1,4 manserint edd. cett. manserunt $\mathbf{v}^{\mathbf{f}} \mathbf{f} \mathbf{z}$

Civ. 1, 86,3 habeant edd. cett. habebant $\mathbf{v}^{\mathbf{f}} \mathbf{f} \mathbf{z}$ 
c) Mediante la introducción de lecturas sin base en la tradición manuscrita o impresa anterior:

En este ámbito cobran la mayor relevancia algunas correcciones de la edición giuntina que constituyen conjeturas atinadas. La edición giuntina recoge algunas correcciones introducidas por primera vez en la edición Giuntina de 1508 que representan una enmienda críticamente plausible y aceptada por los editores modernos:

Es el caso de la corrección diduci por deduci, lectura transmitida por la tradición manuscrita e impresa anterior en Gall. 3, 23,7:

quod ubi Crassus animadvertit, suas copias propter exiguitatem non facile diduci (f z: deduci codd. edd. inc.), hostem et vagari, et vias obsidere, et castris satis praesidii relinquere.

La corrección de $\mathbf{f}$ resulta críticamente pertinente y es adoptada inmediatamente por $\mathbf{z}$. Todos los editores modernos, Du Pontet ${ }^{26}$, Constans, Klotz, Seel y Hering, la asumen.

En la misma dirección se advierte otra corrección significativa introducida por $\mathbf{f}$ y adoptada por $\mathbf{z}$, sin respaldo en la tradición anterior:

\section{Gall. 3, 28,4 desideraverunt f $\mathbf{z}$}

Según Meusel $^{27}$, esta corrección procede de Beroaldus, en su edición de los Commentarii de Lyón 1512; sin embargo, se encuentra por primera vez en la edición florentina de 1508, que además es previa a la de Beroaldus del mismo año, publicada el 20 de junio.

De otro lado existen variantes que constituyen errores separativos respecto al resto de la tradición, que $\mathbf{z}$ también reproduce:

Civ. 1, 87,5 est iter factum edd.inc. $\mathbf{b}$ iter est factum $\mathbf{f} \mathbf{z}$

Civ. 2, 2,3 atque hac agger edd.inc. b atque huc agger $\mathbf{f} \mathbf{z}$

Civ. 2, 6,1 Massiliensibus res nulla edd. inc. b res nulla Massiliensibus $\mathbf{f} \mathbf{z}$

Desde el punto de vista textual, el texto de 1511 mimetiza al de 1508, adoptando todas las variantes de su modelo, y sólo se registran algunos casos en los que ha introducido un error en el proceso de impresión respecto a su modelo:

\section{Civ. 1, 86,2 differretur edd. inc. $\mathbf{f}$ differetur $\mathbf{z}$}

La dependencia textual que $\mathbf{z}$ muestra respecto a su modelo llega al extremo de adoptar sistemáticamente las preferencias gráficas de $\mathbf{f}$, reproduciendo sus vacilaciones ${ }^{28}$, mimetizando el uso de la mayúscula ${ }^{29}$ y su distribución ${ }^{30}$ y sólo excepcionalmente se aparta de su modelo en el uso de la mayúscula:

\footnotetext{
${ }^{26}$ Sin embargo, en la edición oxoniense a cargo de Du Pontet la corrección diduci se atribuye a la edición de Giunta 1514, cuando en realidad se encuentra ya en la de 1508.

${ }_{27}$ H. Meusel, Tabula Coniecturarum, Lexicon Caesarianum, vol. II, Pars II, Berlín, 1893, p. 13.

${ }^{28}$ Aphr. 91,3 aegisset $\mathbf{f} \mathbf{z}$.

${ }^{29}$ Gall. 3, 23,1 vocatiorum ... tarusatium edd. inc. Vocatiorum ... Taurasatium $\mathbf{f} \mathbf{z}$.

${ }^{30}$ Así por ejemplo, $\mathbf{z}$ sigue a su modelo cuando $\mathbf{f}$ prefiere la minúscula: Hisp. 1,1 hispaniae ... italia; 1,5 italiam; 2,1 hispaniam 7,4; africa 8,2; hispaniae; 8,3 africa; 8,5 hispaniae; 10,1 italia.
} 
Gall. 1, 53,8 Marius Metius $\mathbf{f}$ marius Metius $\mathbf{z}$

Gall. 1, 54,3 Labienum f labienum $\mathbf{z}$

Hisp. 14,3 pompeiani f Pompeiani $\mathbf{z}(\mathrm{cf} .16,1)$

\section{DivergenCias ENTRE $\mathbf{z}$ Y $\mathbf{f}$}

Desde el punto de vista textual, la edición veneciana es idéntica a la florentina; sin embargo $\mathbf{z}$ introduce algunos elementos ajenos al propio texto que modifican la composición y le confieren un aspecto distinto:

a) Formato en folio frente al nuevo formato en octavo adoptado por f $\mathbf{f}$, a imitación de las ediciones aldinas, que empieza a utilizar este formato para los textos latinos en el paso al s. $\mathrm{XVI}^{31}$.

b) Caja de escritura de $\mathbf{z}$ es de 45 líneas, con unos 65/70 caracteres por línea, frente a las 30 de $\mathbf{f}$, con 50/55 caracteres por línea.

Para disimular la afinidad entre ambos texto, la edición veneciana tiende a buscar la discoincidencia de fin de línea, si bien es posible advertir varias coincidencias por página, como un mecanismo de control tipográfico durante la composición, dado que los textos son idénticos: algunas de estas coincidencias de fin de línea suceden al final de página de $\mathbf{z}$ :

z (f. 105 r, lín. 45) Hisp. 3,7 ut sileat / verbum facere = f (f. 236v, lín. 25)

$\mathbf{z}$ (f. 105v, lín. 45) Hisp. 7,4 in montibus castra ha / buit posita = f (f. 2337v, lín. 1)

c) Frente a la tipografía en caracteres cursivos de f, siguiendo el modelo aldino, la edición veneciana utiliza la redonda humanística, habitual en el taller de Zannis.

d) Alteraciones en la disposición de los elementos de los títulos de los libros: la edición florentina coloca tras el título la indicación del libro correspondiente, mientras que la edición veneciana lo reproduce pero repite en una primera linea el número del libro:

f A. Hircii autorii commentariorum de bello Hispaniensi Liber sextus (f. 235v)

z Liber sextus A. Hircii autorii commentariorum de bello Hispaniensi liber sextus (f. $105 \mathrm{v})$

e) Utilización ocasional de abreviaturas distintas:

Aphr. 91,2 Ro. Po. f Ro. Popu. z

31 Aldo introduce el formato enchiridii forma y el tipo cursivo en cuerpo pequeño (11) creado por Francesco Griffo por primera vez en la edicion de Virgilio de abril de 1501 (cf. A. Tinto, Il corsivo nella tipografia del Cinquecento, Milán, 1972, pp. 13-43; G. Mardersteig, «Aldo Manzio e i caratteri di Francesco Griffo da Bologna», Studi di bibliografia e di storia in onore di Tammaro de Marinis, vol. III, 1964, pp. 105-47), aunque la primera muestra del nuevo tipo se imprime ya en la Epistole de Sancta Catharina da Siena, fechada en septiembre de 1500, como ya demostró R. Ridolfi, «Del carattere italico aldino nel seculo XV», Scritti sopra Aldo Manuzio, Florencia, 1955, pp. 101-105; la imitación del nuevo formato fue inmediata: Philippus Giunta recurre a él desde la edición de Catulo, Propercio y Tibulo de 1503 (cf. F.J. Norton, Italian Printers, op. cit., pp. 29-30). 
f) $\mathbf{z}$ incorpora otros dos elementos que le otorgan un aspecto muy distinto a su modelo: un grabado al comienzo de cada libro, y tituli marginales, al igual que las ediciones incunables de 1480, 1482 y 1499 , si bien con texto diferente a éstas.

g) La sustitución de la virgula curva de $\mathbf{f}$-que introduce por primera vez la edición florentina para marcar el comma, de acuerdo con innovación tipográfica introducida en el entorno aldino- - por los dos puntos de la tipografía incunable que sigue utilizando $\mathbf{z}$.

Todos estos recursos son habituales en las obras impresas de Augustinus de Zannis y gracias a ellos consigue, en este caso, una edición con una apariencia totalmente distinta a la de su modelo, cuando en realidad no es así.

\section{El TRATAMIENTO DE LA PUNTUACIÓN}

La edición veneciana se apoya completamente en la puntuación de su modelo, si bien modifica el sistema gráfico que había introducido la edición de $1508^{32}$ : en efecto, $\mathbf{z}$ sustituye la vírgula curva empleada en el texto florentino por los dos puntos habituales en las ediciones incunables ${ }^{33}$.

De esta forma $\mathbf{z}$ se basa en la edición florentina pero a la vez recupera el sistema de representación gráfica de la puntuación de los primeros impresos, evidenciando una equiparación completa de la coma semicircular de su modelo con los dos puntos.

Así mismo, $\mathbf{f}$ introduce paréntesis que no están en la tradición anterior y que $\mathbf{z}$ reproduce:

Gall. 2, 1,1 (ita ut supra demonstravimus) ... (quam tertiam ... dixeramus) $\mathbf{f} \mathbf{z}$

La edición florentina había llevado a cabo una tentativa de regularizar el uso de la vírgula curva, frente a la tradición impresa anterior, no sólo para delimitar estructuras dentro de oraciones complejas, sino también para introducir incisos entre sintagmas y construcciones que requieren a juicio del editor una pausa en la entonación a la vez que contribuyen a aclarar su sentido.

Pues bien, $\mathbf{z}$ se ciñe a las propuestas de puntuación de $\mathbf{f}$ pero sustituyendo el signo de coma semicircular por los dos puntos, que se utiliza regularmente en esta edición:

a) Tras verbo en forma personal en oraciones compuestas, o delante del pronombre relativo, para delimitar construcciones sintácticas, que $\mathbf{z}$ sigue:

Gall. 6, 8,8

propinqui Indutiomari, qui defectionis auctores fuerant, comitati eos, ex civitate excesserant $\mathbf{f}$

${ }^{32}$ En efecto, $\mathbf{f}$ es la primera edición de la historia del texto cesariano en la que se introduce sistemáticamente la vírgula curva o coma semicircular para la puntuación débil, -en lugar de los dos puntos para marcar el comma de la tradición impresa anterior- y se generaliza su uso como marca en contraposición al punto bajo como indicio de final de frase o de período, que, por primera vez también, en la tradición del texto cesariano, se emplea sistemáticamente seguida de mayúscula.

${ }^{33}$ Sobre la evolución de la puntuación en las ediciones de este periodo, cf. A. Moreno, «En torno a la puntuación de las primeras ediciones postincunables de los Commentarii de César (1504-1513)», Homenaje a Vicente Picón, Universidad Autónoma de Madrid, Madrid 2007, e.p. 
propinqui Indutiomari: qui defectionis auctores fuerant: comitati eos: ex defectione excesserant $\mathbf{z}\left(\mathbf{t} \mathbf{v}^{\mathbf{s}} \mathbf{v}^{\mathbf{z}}\right)$

propinqui Indutiomari: qui defectionis auctores fuerant comitati eos ex civitate excesserant $\mathbf{v}$

$\mathbf{z}$ sigue a $\mathbf{f}$ frente a las ediciones anteriores, sobre todo cuando $\mathbf{f}$ ha introducido la marca de comma en diferentes contextos:

a) Para delimitar construcciones de ablativo:

Gall. 6, 10,2 his cognitis rebus, rem frumentariam providet $\mathbf{f}$ (... rebus: rem... z) his cognitis rebus rem frumentariam providet $e d d$. inc. $\mathbf{b}$

Gall. 6, 7,4 Labienus hostium cognito consilio, sperans temeritate eorum fore aliquam dimicandi facultatem $\mathbf{f}$ (... consilio: sperans... z $)$

Labienus hostium cognito consilio sperans temeritate eorum fore aliquam dimicandi facultatem edd. inc. b a

Civ. 2, 10,7 subitoque inopinantibus hostibus machinatione navali, phalangis subiectis, ad turrim hostium admovent $\mathbf{f}$ (... subiectis: ad... $\mathbf{z}$ ) subitoque inopinantibus hostibus machinatione navali phalangis subiectis ad turrim hostium admovent $\mathbf{v} \mathbf{b} \mathbf{v}^{\mathbf{s}} \mathbf{v}^{\mathbf{s}} \mathbf{b}$ subitoque inopinantibus hostibus machinatione navali phalangis: subiectis ad turrim hostium admovent $\mathbf{t}$

b) En la coordinación de sintagmas, delante de et, ac, atque, neque:

Gall.3, 29,2 cum iam pecus, atque extrema impedimenta a nostris tenerentur f (... pecus: atque... z $\mathbf{z}$ ) cum iam pecus atque extrema impedimenta a nostris tenerentur $e d d$. inc. $\mathbf{b}$

c) Entre sintagmas coordinados con -que:

Gall. 3, 29,3 ita vastatis omnibus eorum agris, vicis, aedificiisque incensis $\mathbf{f}$ a ita vastatis omnibus eorum agris: vicis: aedificiisque incensis: $\mathbf{z}$ ita vastatis omnibus eorum agris: vicis aedificiisque incensis: edd. inc. $\mathbf{b}$

Gall. 6, 7,4 cum viginti quinque cohortibus, magnoque equitatu f a cum viginti quinque cohortibus: magnoque equitatu $\mathbf{z}$ cum viginti quinque cohortibus magnoque equitatu edd. inc. b

Gall. 6, 7,6 dicantur, sese suas, exercitusque fortunas $\mathbf{f}$ a dicantur: sese suas: exercitusque fortunas $\mathbf{z}$ dicuntur, sese suas exercitusque fortunas edd. inc. $\mathbf{b}$

Gall. 6, 7,8 tribunis militum, primisque ordinibus convocatis $\mathbf{f}$ tribunis militum: primisque ordinibus convocatis $\mathbf{z}$ tribunis militum primisque ordinibus convocatis edd. inc. $\mathbf{b}$

Gall. 6, 8,6 ac primo concursu in fugam conversi, proximas silvas petiverent $\mathbf{f}$ ac primo concursu in fugam conversi: proximas silvas petiverent $\mathbf{z}$ ac primo concursu in fugam conversi proximas silvas petiverent edd. inc. $\mathbf{b} \mathbf{a}$ 


\section{Gall. 6, 11,3 summa omnium rerum, consiliorumque redeat $\mathbf{f}$ a summa omnium rerum: consiliorumque redeat $\mathbf{z}$ summa omnium rerum consiliorumque redeat $e d d$. inc. b}

Pero $\mathbf{z}$ se atiene a su modelo incluso en el caso inverso, es decir, cuando $\mathbf{f}$ prescinde de la notación de la coma cuando ésta se encuentra presente en toda la tradición anterior:

Gall. 6, 8,1

tam exiguam manum: praesertim fugientem: atque impeditam adoriri non audeant edd. inc. a

tam exiguam manum praesertim fugientem, atque impeditam adoriri non audeant $\mathbf{f}$ (... fugientem: atque... z $\mathbf{z}$ )

Cuando $\mathbf{f}$ altera la puntuación de la tradición incunable, $\mathbf{z}$ siempre le sigue:

\section{Gall. 323,1}

Armis obsidibusque acceptis: Crassus in fines vocatium et tarusatium profectus est. tum vero barbari $\mathbf{r} \mathbf{t} \mathbf{v}^{\mathbf{s}} \mathbf{v}^{\mathbf{f}}$

Armis obsidibusque acceptis Crassus in fines vocatium et tarusatium profectus est. tum vero barbari $\mathbf{v}$

Armis obsidibusque acceptis Crassus, in fines Vocatiorum et Tarusatium profectus est. tum vero barbari $\mathbf{f}$

Armis obsidibusque acceptis Crassus: in fines Vocatiorum et Tarusatium profectus est. tum vero barbari $\mathbf{z}$

En este caso, $\mathbf{f}$ ha desplazado la coma tras Crassus y $\mathbf{z}$ le sigue. La edición aldina recupera la puntuación de los incunables frente a la edición de 1508.

Una muestra inequívoca de esta situación se verifica en algunas innovaciones de la puntuación de $\mathbf{f}$ que $\mathbf{z}$ asume frente a toda la tradición anterior y que esclarecen la sintaxis de un pasaje:

Gall. 6, 12,6

adventu Caesaris facta commutatione rerum, obsidibus redditis, veteribus clientibus restitutis: novis per Caesarem comparatis $\mathbf{f}$ adventu Caesaris facta commutatione rerum: obsidibus redditis: veteribus clientibus restitutis: novis per Caesarem comparatis $\mathbf{z}$

En este caso $\mathbf{f}$ revisa las relaciones sintácticas entre esta sucesión de ablativos, suprimiendo la coma inicial tras Caesaris de la primeras ediciones, pero la añade detrás de rerum; de esta forma $\mathbf{f}$ sugiere una forma de leer el pasaje en virtud de la cual adventu Caesaris no está coordinado con commutatione sino que depende de éste, propuesta que los editores críticos modernos siguen en la puntuación.

En cambio en este pasaje las primeras ediciones incunables $(\mathbf{r} \mathbf{v} \mathbf{t})$ inducen a interpretar la sucesión de ablativos al mismo nivel sintáctico, marcando cada construcción de ablativo mediante los dos puntos del comma: 
adventu Caesaris: facta commutatione rerum obsidibus redditis: veteribus clientibus restitutis: novis per Caesarem comparatis... r v t b

Las ediciones posteriores $\left(\mathbf{v}^{\mathbf{s}} \mathbf{v}^{\mathbf{f}}\right)$ muestran una mayor vacilación al prescindir de esta marca en los primeros sintagmas e incluirla en los últimos de la serie:

adventu Caesaris facta commutatione rerum obsidibus redditis: veteribus

clientibus restitutis: novis per Caesarem comparatis... $\mathbf{v}^{\mathrm{s}} \mathbf{v}^{\mathbf{f}}$

En la misma línea se inscribe el caso de Gall. 6, 10,1, donde f introduce la virgula curva para delimitar con claridad la entonación y los límites de la oración de relativo y distinguirla del infinitivo denuntiare:

fit certior...atque his nationibus: quae sub eorum sunt imperio denuntiare, edd. inc. a

fit certior... atque his nationibus, quae sub eorum sint imperio, denuntiare, $\mathbf{f} \mathbf{z}$

Los editores críticos modernos siguen la puntuación que propuso f, edición que revela una intención clara de concebir este recurso como un soporte para la interpretación de los textos.

Sólo excepcionalmente $\mathbf{z}$ altera la puntuación de $\mathbf{f}$ :

Aphr. 91,2 quod bello contra populum Romanum suscepto, in oppido Zamae $\mathbf{f}$ quod bello contra populum Romanum suscepto in oppido Zamae $\mathbf{Z}$

Aphr. 91,3 postquam Iuba ante portas diu multumque primo minis $\mathbf{f}$ postquam Iuba ante portas diu, multumque primo minis $\mathbf{z}$

Hisp. 15,6 nostro hospites, qui in oppido erant, iugulare, et de muro $\mathbf{f}$ nostro: hospites: qui in oppido erant: iugulare: et de muro $\mathbf{z}$

En el pasaje que sigue $\mathbf{z}$ introduce un punto sin base en su modelo y que constituye un claro ejemplo de error inducido por la mayúscula que sigue:

Aphr. 91,3 uti se ad suos Deos penates admitterent $\mathbf{f}$

uti se ad suos. Deos penates admitterent $\mathbf{z}$

\section{Conclusiones}

La edición de los Commentarii de César impresa en 1511 en los talleres de Augustinus de Zannis de Portesio (z) se presenta aparentemente como un producto típico de los hábitos filológicos de la época, caracterizados por la práctica de la emendatio humanística, a tenor del título y el colofón de la obra y del prefacio de Lucas Olchinensis, en el cual este filólogo y jurista italiano afirma que ha preparado la edición con denuedo mediante la enmienda de las numerosas corrupciones que presentan las ediciones anteriores. 
Sin embargo, el análisis crítico de esta edición y su colación con las ediciones precedentes del entorno italiano permite comprobar que el texto de $\mathbf{z}$ depende de manera exclusiva y completa de la edición florentina de 1508 (f), impresa por Philippus Giunta y preparada por Lucas Robia. Aunque la apariencia editorial es completamente distinta, $\mathbf{z}$ adopta todas las lecturas de su modelo, incluyendo aquellas en las que la edición giuntina ha incorporado variantes de los mss. ajenas a la tradición impresa anterior así como otras exclusivas que responden a correcciones propias y de gran valor crítico (como diduci por diduci de Gall. 3, 23,7) que muestran que esta edición sí que responde, en cambio, a una emendatio ope codicum atque ingenii, de la cual es totalmente deudora la edición veneciana de 1511, que de Zannis volvió a editar en 1517.

Esta identidad textual es absoluta entre ambas ediciones y revela que en $\mathbf{z}$ no subyace la labor filológica de corrección proclamada por el impresor y por el presunto editor de la misma, sino una reproducción mecánica del texto de su modelo f, reproducción que afecta a todos los niveles textuales (selección de variantes, grafías, puntuación...) y que es ejecutada escrupulosamente por el taller veneciano, pero que sin embargo se intenta soslayar a través de una composición de imprenta totalmente distinta y que transmite la apariencia de que estamos ante dos obras distintas: en efecto, mientras la edición giuntina responde a la imitación del nuevo formato aldino del libro de bolsillo, en octavo y con tipografía cursiva de cuerpo pequeño, la edición veneciana presenta el aspecto de los antiguos incunables, con formato en folio y tipo romano convencional, así como mediante otros detalles editoriales con los que se intenta diferenciar, como la introducción de xilografías al comienzo de cada libro y títulos marginales, ausentes en la edición florentina y, sobre todo, por la sustitución de la vírgula curva para marcar el comma que utiliza f por el antiguo signo de los dos puntos, habitual en la tradición incunable y que además viene a probar la conciencia de la equivalencia entre ambos signos gráficos.

Así pues, esta edición veneciana no es lo que en términos de Severyns ${ }^{34}$ se entiende como una edición primaria, sino una edición secundaria pero sin las correcciones fruto de algún proceso de emendatio que suele caracterizar a esta modalidad de ediciones humanísticas. De esta forma, Augustinus de Zannis ofrece, bajo la apariencia de las antiguas ediciones incunables habituales en su taller, un texto que en realidad es el que tres años antes había preparado Lucas Robia para la imprenta florentina de Philippus Giunta.

\section{APÉNDICE: DESCRIPCIÓN DE LA EDICIÓN}

Título: Caii Julii Caesaris: Invictissimi īperatoris / cōmentaria: seculorum iniuria antea difficilia: et valde mendosa. Nunc / primum a viro docto expolita: et optime recognita. Additis / de novo apostillis: Vna cū figuris suis locis apte / dispositis. Nec non regulata tabula quae omnia / loca: flumina: montes: urbes: op/pida: ifinita prelia: et quaeque digna cognitu mirifice demōstrat.

${ }^{34}$ A. Severyns (Texte et apparat, op. cit. pp. 7-68) distingue entre ediciones primarias (las que están preparadas por el editor a partir de fuentes mss. medievales), y secundarias (en las cuales el editor trabaja sobre una edición impresa previa sobre la cual introduce sus enmiendas). 
Colofón: f. $\mathrm{p}_{6 \mathrm{v}}(110 \mathrm{v})$ : Habes lector peritissime cōmētaria ante impressorum in/iuira trunca: et mendosa: Nunc autem a viro docto optime re/cognita: et in pristinum candorem redacta: Impressa mira / diligentia Venetiis: per Augustinū de Zannis de / Portesio Anno reconciliatae nativitatis/.M.D.XI. die .XVII. Augusti.

Descripción física: 4 ff. sin numerar; 110 ff. numerados y 8 ff. sin numerar.

$$
\text { sign.: } \mathrm{a}_{4}, \mathrm{~b}_{8}-\mathrm{b}_{8}, \mathrm{~g}_{10}, \mathrm{~h}_{8}-\mathrm{n}_{8}, \mathrm{o}_{6}, \mathrm{p}_{6}, \mathrm{q}_{8}
$$

En folio, caracteres romanos. Título en gótica. 45 Lineas por pag. (42 según Sander).

Contiene varias xilografías: una grande en la portada, en rojo y negro; ésta aparece repetida pero en negro en $b_{1}$; y doce pequeñas al comienzo del resto de los libros. Algunos de estos grabados proceden de la edición de Tito Livio de 11 de febrero de 1493 (Essling: II, I p. 23).

Contenido: $\mathrm{f} . \mathrm{a}_{1}$ Lucas olchinensis: artium et legum professor: Rosello de rosellis patavo foelicitatem; f. $a_{2}$ Commentariorum Caesaris Tabula; f. $b_{1}$ Liber primus de bello Gallico; f. $c_{1}$ Liber secundus; f. $c_{5}$ Liber tertius; f. $c_{8 v}$ Liber quartus; f. $d_{5}$ Liber quintus; $f$. $e_{5}$ Liber sextus; $f$. $f_{1 v}$ Liber septimus; $f$. $g_{4 v}$ Liber octavus; $f$. $h_{1}$ Liber primus de bello Civili; f. $i_{4}$ Liber secundus; $f$. $k_{2}$ Liber tertius; $; f$. $l_{8 v}$ De bello Alexandrino; $f . n_{2 v}$ De bello Africo; f. $\mathrm{p}_{1}$ Liber sextus...de Bello Hispaniensi; ff. $\mathrm{q}_{1 \mathrm{r}}-\mathrm{q}_{8 \mathrm{r}}$ Index commentariorum C. Iulii Caesaris: \& earum rerum ... Raymundus Marlianus inuenit atque addidit; f. $\mathrm{q}_{8 \mathrm{v}}$ en blanco.

Descripciones anteriores: Essling ${ }^{35}$ 1727; Isaac ${ }^{36}$ 12447; Index Aur $^{37}$. 128.655; Adams $^{38}$ C-28; Sander $^{39}$ 1503; Méndez Aparicio ${ }^{40}$, 1580; ICCU C125; CNCE 8146 (ed. de 1511) y 8149 (ed. de 1517).

\section{Ejemplares conservados en España:}

a) Edición de 1511:

— Los dos únicos ejemplares conservados se encuentra en Cáceres, Biblioteca Pública «A. Rodríguez Moñino y María Brey», sign. 1/4557 sin enc. (olim: A/4557 -

${ }^{35} \mathrm{P}$. Essling, Les livres à figures vénitiens de la fin du XV siècle et du commencement du XVI. Florence-Paris, 1907-1914

${ }^{36}$ F. Isaac, Index to the Early Printed Books in the British Museum, pt. II, 1501-1520. sect. II (Italy), Londres, 1938, 12447.

37 Index Aureliensis. Tertia Pars, Tomus III: Clavis typographorum librariorumque saeculi sedecimi, Baden Baden, 1992.

${ }^{38}$ H. M. Adams, Catalogue of Books Printed on the Continent of Europe, 1501-1600, in Cambridge Libraries, Londres, 1967, p. 219 (edición de 1517, n. ${ }^{\circ}$ 28). Cf. también Short-title catalogue of books printed in Italy and of Italian books printed abroad 1500-1600, Boston, 1970, vol. I, p. 320; P. Kristeller, Die italienischen Buchdrucker- und Verlegerzeichen bis 1525, Strassburg, 1893 (reimpreso en Mansfield Center CT, 2000).

${ }_{39}$ M. Sander, Le livre à figures italien depuis 1467 jusqu’à 1530, Milán, 1942, vol. I, pp. 274-275, n 1503.

40 J. A. Méndez Aparicio, Catálogo de los impresos del siglo XVI de la Biblioteca pública del estado. Toledo, 3 vol. 1993-1996 (ed. de 1517). 
R. 8071) CCPB000455063-3; y sign. 1/8888(1) - Enc. mudejar - (olim: A/8888 R. 7168) CCPB000456367-0.

b) Edición de 1517 (CCPB000029170-6):

- Toledo, Biblioteca de Castilla-La Mancha, Fondo Antiguo, Res. 308 y M0253(2).

— Orihuela, Biblioteca Pública «Fernando de Loazes», Fondo Antiguo, R. 10162 (2).

- Salamanca, Universidad de Salamanca. Biblioteca General Universitaria, 34568.

Existen ejemplares de estas ediciones en diversas bibliotecas europeas ${ }^{41}$. En el caso italiano, El Censimento delle edizioni italiane del XVI secolo que se viene desarrollando en el Istituto Centrale per il Catalogo Unico delle biblioteche italiane e per le informazioni bibliografiche (ICCU http://edit16.iccu.sbn.it/web_iccu/ihome.htm) ${ }^{42}$, identifica los ejemplares de esta obra en Italia, de la edición de 1511 (CNCE 8146) ${ }^{43}$ y de la de 1517 (CNCE 8149) ${ }^{44}$.

${ }^{41}$ Entre ellos, un ejemplar se conserva en París, BNF, Tolbiac - Rez-de-jardin, sign. RES 4- Z ADLER- 13, y en Londres, British Library, sign. IB.24059/3.

${ }^{42}$ Hasta el momento se han publicado 5 volúmenes: La edizioni italiane del XVI secolo: censimento nazionale, que desde 1985 se publica en Roma (V vol., 2005).

${ }^{43}$ BG0026 Biblioteca civica A. Mai - Bergamo; FC0021 Biblioteca del Seminario vescovile - Forlì; FE0017 Biblioteca comunale Ariostea - Ferrara; FI0098 Biblioteca nazionale centrale - Florencia; MI0185 * Biblioteca nazionale Braidense - Milán; NA0079 Biblioteca nazionale Vittorio Emanuele III - Nápoles; NO0054 Biblioteca comunale Carlo Negroni - Novara; PT0025 Biblioteca comunale Forteguerriana - Pistoia; RM0267 Biblioteca nazionale centrale - Roma; RM0290 Biblioteca Angelica - Roma; RM0313 Biblioteca Casanatense Roma; RN0028 Biblioteca comunale Antonio Baldini - Santarcangelo di Romagna; SI0046 Biblioteca comunale degli Intronati - Siena; VE0039 Biblioteca d'arte del Museo civico Correr - Venecia; VE0049 Biblioteca nazionale Marciana - Venecia.

${ }^{44}$ AP0012 Biblioteca comunale - Fermo; BO0304 Biblioteca comunale dell'Archiginnasio - Bolonia; BS0124 Biblioteca comunale G. U. Lanfranchi - Palazzolo sull'Oglio; GE0038 Biblioteca universitaria - Génova; MI0133 Biblioteca Ambrosiana - Milán; NA0079 Biblioteca nazionale Vittorio Emanuele III - Nápoles; PC0030 Biblioteca comunale Passerini Landi - Piacenza; PD0158 Biblioteca universitaria di Padova - Padua; PR0072 Biblioteca Palatina - Parma; RA0016 Biblioteca comunale Manfrediana - Faenza; RA0036 * Biblioteca comunale Classense - Ravenna; RI0120 Biblioteca diocesana - Rieti; RO0049 Biblioteca dell'Accademia dei Concordi - Rovigo; TO0240 Biblioteca civica centrale - Turín; TR0037* Biblioteca del Seminario vescovile - Amelia; UD0123 Biblioteca P. Bertolla del Seminario arcivescovile - Udine; VE0047 Biblioteca Querini Stampalia - Venecia; VE0049 Biblioteca nazionale Marciana - Venecia; VI0101 Biblioteca del Seminario vescovile - Vicenza; VR0061* Biblioteca del Seminario vescovile - Verona; VR0070 Biblioteca dell'Accademia filarmonica di Verona - Verona. 